Revista Calidad en la Educación Superior

Programa de Autoevaluación Académica

Universidad Estatal a Distancia, Costa Rica

ISSN 1659-4703

revistacalidad@uned.ac.cr

\title{
JORNADAS EMPRENDEDORAS: GENERANDO ESPACIOS PARA IDEAS EMPRENDEDORAS DE LA POBLACIÓN ESTUDIANTIL DE UNED
}

\author{
ENTREPRENEURS JOURNALS: GENERATING IDEAS FOR ENTREPRENEURS \\ SPACES BYTHE STUDENT POPULATION Of UNED
}

\author{
Eduardo Jirón-Fajardo ${ }^{1}$ \\ ejiron@uned.ac.cr \\ Helene Giselle Chan-Castillo ${ }^{2}$ \\ hchang@uned.ac.cr \\ Universidad Estatal a Distancia, Costa Rica
}

\author{
Volumen 7, Número 2 \\ Noviembre 2016
}

pp. $203-230$

Recibido: 25 de setiembre, 2016

Aprobado: 31 de octubre, 2016

\footnotetext{
1 Licenciatura en Ingeniería Industrial, Universidad de Costa Rica, Post-grado en Gerencia de Operaciones, INCAE; Magister en Administración de Empresas, INCAE; Diplomado en Gestión Internacional, Instituto de Empresas de Madrid, España. Profesor de la Escuela de Ciencias de la Administración-UNED y Director de la Cátedra de Producción. Correo electrónico: ejiron@uned.ac.cr

2 Bachillerato y Licenciatura en Administración Pública, Universidad de Costa Rica, Magister en Dirección de Empresas con énfasis en Gerencia, Universidad de Costa Rica, estudios realizados sin concluir en Doctorado en Dirección de Empresas Estrategias y Organización, Universidad de Valencia, España. Directora de Carrera Administración de Empresas con énfasis en Negocios Internacionales, Universidad Estatal a Distancia, Costa Rica. Correo electrónico: hchang@uned.ac.cr
} 


\title{
Resumen
}

Las "Jornadas Emprendedoras" son el desarrollo de actividades que ayuden a generar un ambiente que facilite emprendimientos en la población estudiantil de la Universidad Estatal a Distancia (UNED). De esta manera, se aprovecha el esfuerzo de un trabajo académico realizado por los estudiantes para enfocarlo como una potencial solución al desempleo en diversas zonas del país donde se necesite. En esta primera experiencia académica, durante el primer cuatrimestre del año 2016, se incorporaron estudiantes de diferentes centros universitarios y carreras de la Escuela de Ciencias de la Administración (ECA) principalmente. En los 11 proyectos, se evidencia el trabajo colaborativo entre estudiantes, apoyándose en su aprendizaje sobre estudios de pre-factibilidad, cuya importancia radica en su carácter multidisciplinario. Se logra visualizar una propuesta de vinculación universidad-empresa, en donde el interés por parte de los estudiantes es innovar y generar ideas de negocios. La experiencia del proyecto de "Jornadas Emprendedoras", demuestra la importancia de extender este tipo de actividades a toda la población estudiantil de la UNED. Este tipo de espacios e instrumentos académicos se visualizan para ser incluido dentro de los Trabajos finales de graduación, Comisión de Investigación Extensión de la ECA (COMIEX-ECA), inclusive otras carreras que no pertenecen a la ECA.

Palabras clave: Emprendedurismo; Gestión del conocimiento; Integración empresarial (alianzas; cooperación); Vinculación universidad-empresa; Trabajo colaborativo.

\begin{abstract}
"Entrepreneurs Journals" is the development of activities that help creating an environment that facilitates entrepreneurship in the students of Universidad Estatal a Distancia (UNED). Thus, the effort of the students' academic work is taken advantage of, and is focused as a potential solution to unemployment in certain areas of the country; this kind of initiatives might generate employment. In this first academic experience during the first term of 2016, students from different University Sites and careers of the School of Management Sciences were incorporated. In the eleven projects there is evidence of collaborative working among the students, relying on their learning about pre-feasibility studies and, the most important part, being multidisciplinary. It shows a proposal of university-company association, where the students' interest is to innovate and generate business ideas. The experience of the"Jornadas Emprendedoras" project shows the importance of extending its research instruments to the entire student population at UNED: Final Graduation Projects, Research and Extension Committee, and even other careers.
\end{abstract}

Keywords: Entrepreneurship; Knowledge Management; Business Integration (alliances; cooperation); university- enterprise; collaborative work. 


\section{Introducción}

Como facultad de negocios, la Escuela de Ciencias de la Administración (ECA) de la UNED en Costa Rica, ha de desarrollar iniciativas y actividades que genere un ambiente para facilitar emprendimientos en la población estudiantil. El presente proyecto explora el potencial implícito para una universidad basada sobre un modelo a distancia, donde se integran estudiantes, personal académico y los centros universitarios en sus comunidades.

Dichos emprendimientos estudiantiles en el largo plazo se ven como oportunidades de desarrollo económico para comunidades, donde se concreten ideas de negocios y así volverse generadores de empleo. La economía nacional está compuesta por distintos sectores industriales con sus diferentes realidades, donde la universidad ha de enfocar esfuerzos que faciliten la ignición de ambientes emprendedores en distintas zonas del país.

El emprendedurismo es uno de los ejes transversales de la ECA, por lo que se deben brindar espacios académicos abiertos para promover emprendimiento 0 creación de empresas entre la población estudiantil. A largo plazo, se visualiza oportunidades de desarrollo económico en comunidades donde se logre concretar algunos emprendimientos estudiantiles que generen empleo. Se requiere un catalizador que facilite ese ambiente generador y propicie el aporte intelectual, combinado con necesidades en las comunidades donde residen los estudiantes, y así, concretar las oportunidades de negocios en un contexto académico.

El objetivo general de la práctica es incentivar el desarrollo de emprendimientos en estudiantes de UNED. Y sus objetivos específicos son:

Dinamizar la creación de proyectos con la finalidad de ejecutarlos como empresas. Brindar a los estudiantes de la ECA la oportunidad que se desarrollen dentro de un entorno de aprendizaje apoyado con iniciativas de emprendimiento.

Generar mayor interacción entre tutores y estudiantes, así como la conformación de redes de estudiantes interdisciplinarios dentro UNED. 
La metodología que se utiliza en la investigación es de tipo mixto. Entre los resultados arrojados está el hecho de que los estudiantes participantes se motiva que se les valore sus ideas para participar y además recibir información útil directamente de emprendedores exitosos en sus negocios.

\section{Contextualización de la actividad}

\section{Reseña sobre la Universidad Estatal a Distancia (UNED)}

La UNED fue creada "como una institución de educación superior especializada en la enseñanza a través de medios de comunicación social" (Artículo 1), mediante Ley No. 6044, publicada en La Gaceta No. 50 del 12 de marzo de 1977. La expansión geográfica y las aspiraciones por el acceso a nuevos sectores de la población a los bienes de cultura que ofrece la universidad son el marco que conlleva a la creación de la UNED, esto producto por el aumento de la población de jóvenes que querían seguir una educación universitaria (UNED, 2004).

El modelo pedagógico de la UNED es un proceso de aprender y ensañar a distancia, este modelo está enfocado en que [...] la comunidad universitaria deberá estar comprometida en su construcción, mediante un proceso dinámico y ampliamente participativo (UNED, 2004).

Luego de 40 años de la creación de esta institución, ha venido a democratizar la educación superior pública. Cuenta con 38 centros universitarios, divididos por regiones (Región Chorotega, Región Huétar Norte, Región Huétar Atlántica, Región Central, Región Pacífico Central, Región Brunca). Este modelo no presencial cubre gran parte de la población estudiantil a nivel regional que no estaba cubierta, la cual varía entre un centro universitario y otro. Al 1er cuatrimestre del 2016, la UNED tiene un total de 21506 estudiantes, 13947 son mujeres y 7559 hombres, (CIEl, 2016). Específicamente en la ECA, la población estudiantil es en total 6179 estudiantes (3 916 son mujeres y 2263 Hombres). La mayor parte de la población estudiantil, a nivel general en la UNED y en las Carreras de la ECA, son mujeres, y la tendencia se sigue manteniendo en los centros universitarios para el I cuatrimestre del 2016. Así que, al promover un ambiente de emprendimiento, 
implica que también se están generando espacios para oportunidades entre la población de estudiantes mujeres de la universidad en las distintas regiones del país.

Figura 1. Centros Universitarios de la UNED por regiones socioeconómicas a lo largo del territorio costarricense.

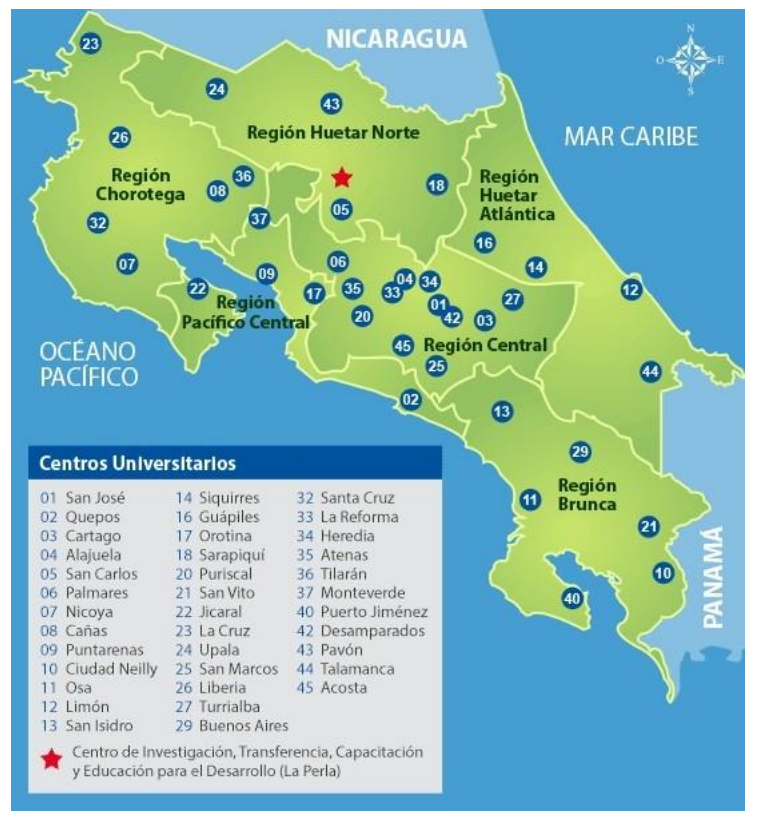

Fuente: www.uned.ac.cr/centros-universitarios

En la figura 1, se muestra el mapa de Costa Rica por Regiones y la distribución de los Centros Universitarios alrededor de todo el país. Desde el año 2008, la ECA define siete ejes transversales que son parte de la fundamentación teóricametodológica de las carreras, los cuales se mencionan a continuación: Ambiente, Derechos humanos, Diversidad cultural, Equidad de género, Investigación, Emprendedurismo y Ética.

Es así que la iniciativa de las "Jornadas Emprendedoras" viene a ser un pilar fundamental en el quehacer académico, que se puede visualizar dentro del plan estratégico y ejes transversales; estas actividades buscan desarrollar y promover el pensamiento creativo, el sentido de autorrealización personal, tanto como, facilitar 
un medio para el uso de las destrezas necesarias en el análisis y evaluación de las condiciones cambiantes del entorno.

\section{Algunos conceptos que contextualizan este estudio}

El concepto de focalizar una idea para el desarrollo y creación de una empresa, viene a jugar un papel fundamental en el análisis teórico sobre los emprendimientos en los negocios y se puede identificar en varias aristas. El emprendedurismo, como eje transversal, incorpora principios que son incluidos dentro de la visión y misión de la ECA. El concepto de "la creación de empresas y la promoción del emprendedurismo", según lo menciona Cabello, C. (2006) se entiende como:

"el proceso de identificar, desarrollar y dar vida a una visión, que puede ser una idea novedosa, una oportunidad o simplemente una mejor manera de hacer las cosas; y cuyo resultado final es la creación de una nueva empresa, formada bajo condiciones de riesgo y considerable incertidumbre"

Según (Drucker, 1985) lo enfoca en la práctica de la innovación, "la innovación es la herramienta básica de los emprendedores, el medio por el cual ellos explotan el cambio como una oportunidad para un negocio o servicio diferente".

Los emprendimientos pueden plantearse desde una perspectiva de necesidades en una comunidad, con el propósito de desarrollar nuevos productos, que por un lado ayuden a generar nuevas tecnologías, diversificar algún producto e innovar, generando un nuevo conocimiento que puede enfocarse en necesidades propias de una región. La vinculación entre la empresa y la universidad, y el modelo de la Triple hélice, plantea un nuevo paradigma que propicia una misión emprendedora para la universidad. A partir de este enfoque la noción de universidad emprendedora se ha desarrollado con mucha más importancia, utilizando avances para el desarrollo socio-económico, actividades de patentes, y licencias e institucionalización de actividades spin-off. Esto ha producido un cambio de actitud entre los académicos con respecto a la colaboración con la empresa. Los nuevos arreglos sociales y canales de interacción son necesarios, si la industria y el gobierno están unidos por las universidades en economías basadas en el 
conocimiento (Etzkowitz, De Mello, 2003). La aparición de la universidad-la empresa- y las interacciones del gobierno también puede ser identificado como un factor clave en el desarrollo regional (Etzkowitz, Klofsten, 2005).

El cambio tecnológico y la cooperación empresarial con las universidades, es un aspecto que ha tomado mayor auge en los últimos tiempos. La competitividad en los negocios y la necesidad de establecer alianzas para lograr mayor participación, tanto en el mercado como en la creación de fuentes de innovación, provoca una nueva proyección de la misma universidad para la investigación y desarrollo de un país. Dentro de la literatura sobre emprendedurismo, cabe señalar tres categorías: emprendedurismo social, emprendedurismo ecológico y empresas de base tecnológica.

De acuerdo con la definición de emprendedurismo social (Zemler, 2008) citado por Araya (2013): "Una práctica que responde a las necesidades de la sociedad y a los vacíos del mercado, con propuestas innovadoras y financieramente sostenibles, diseñadas para resolver problemas sociales. Debe emerger del nexo y cooperación entre los sectores públicos, privados y de organizaciones sin fines de lucro" (p.6). Por otro lado se define el emprendedurismo ecológico (Delgadillo y Alburquerque, 2010), indican que "se busca identificar nuevas oportunidades empresariales que repercutan en la generación de empleos para incrementar los ingresos de la población rural, y los de los habitantes que radican en las aéreas de influencia socioeconómica "[...]. , consiste en convertir los espacios rurales históricamente despoblados adyacentes a las áreas naturales protegidas, en territorios donde es posible el impulso de nuevos emprendimientos de base ecológica, con la adecuada promoción de planes de desarrollo sostenible, donde los actores locales aprovechen las potencialidades territoriales como un activo estratégico para el desarrollo económico de sus propios lugares."

A como dice (Merino y Villar, 2007), en palabras de Palacios, Del Val y Casanueva (2005) "en un entorno cada vez más globalizado y competitivo, el uso de la tecnología como base del desarrollo empresarial resulta ser uno de los mecanismos 
más eficaces para el desarrollo económico de las regiones". "Empresas de Base Tecnológica" (Simón, 2003) citado por Mora y March (2007), es un término que se refiere a "organizaciones productoras de bienes y servicios, comprometidas con el diseño, desarrollo y producción de nuevos productos y/o servicios de fabricación innovadores, a través de la aplicación sistemática de conocimientos técnicos y científicos". (p.99) El contexto actual puede favorecer la creación de nuevas empresas, ya sea por aspectos de cambio tecnológico, el conocimiento, los servicios con tendencia a crecer, problemática de sostenibilidad, las alteraciones en la estructura demográfica y otras más que generan creación de nuevas oportunidades de negocios y empresas, por ende, nuevas fuentes de empleo y movilidad de unos hacia otros empleos.

\section{Las "Jornadas Emprendedoras"}

El propósito principal de las Jornadas Emprendedoras lo expresa su eslogan: "Generando espacios para ideas emprendedoras de la población estudiantil"; además, ya como una investigación, interesa también evaluar ese ambiente de emprendimientos que se genera entre los estudiantes, a raíz de su realización. En síntesis, la actividad se trata de involucrar a estudiantes avanzados (último cuatrimestre del bachillerato o licenciatura) de una carrera, y vincularlos con la realidad de crear una empresa, a partir de una idea de negocio. Por otro lado, es el acercamiento, práctico y guiado por parte de una cátedra (profesores) en el proceso de aprendizaje que realizan los estudiantes.

El contexto real en que están las universidades en Costa Rica es la falta de nuevas fuentes de empleo. Los actuales empleadores no absorben más profesionales recién graduados. En diversos cantones del país, los índices de progreso social (IPS) son variados y algunos evidencian la falta de oportunidades brindadas, que resulta en poco progreso social. "EI IPS es un modelo integral que se utiliza para medir el desarrollo humano, [...] considera tres dimensiones: Necesidades Humanas Básicas, Fundamentos de Bienestar, y oportunidades" (CLADS, 2016). Por tanto, es importante que una escuela de negocios promueva el ambiente de emprendimientos. La iniciativa nace para que las ideas de estudiantes, al realizar 
sus proyectos para cumplir un requisito académico, también sean compartidas con otras personas, dentro y fuera de UNED, y ser exploradas como potenciales soluciones de catalizadores económicos, ya que pretende que el producto final de un determinado proceso de aprendizaje del estudiante se transforme en algo útil para la sociedad.

\section{Primera experiencia "un pequeño plan piloto"}

En la oferta del 1er cuatrimestre en la asignatura Preparación y Evaluación de Proyectos (cód. 535), que forma parte de la malla curricular de varias carreras de la ECA y como parte de la estrategia de aprendizaje de la asignatura, se tiene previsto la realización de un proyecto (grupal) como parte del modelo de evaluación (2 pruebas ordinarias de $30 \%$ cada una y el proyecto con peso $40 \%$ ).

El proyecto (partes a y b) se realiza como trabajo colaborativo entre estudiantes, quiénes construyen el mismo con información real tomada del entorno y apoyándose en conceptos teóricos estudiados. En las orientaciones académicas se indica a los estudiantes (p.7 de orientaciones al estudiante - 1er cuatrimestre, 2016) la importancia de poner en práctica sus habilidades personales de trabajo en equipo.

La falta de oportunidades de empleo en diversas zonas del país requiere que los estudiantes piensen en estas iniciativas que le generen la oportunidad de autoemplearse o volverse empleadores. Entonces, se desarrolla un pequeño piloto con estudiantes y sus proyectos (asignatura 535) del Centro Universitario de Liberia (parte de Región Chorotega en zona norte del país).

Se conversó con los estudiantes que asistían a tutorías del Centro, sobre la intención de las jornadas emprendedoras, quienes le dieron acogida a la iniciativa. Luego se coordinó con la administradora de dicho centro para la logística de la actividad, quien reconoció lo importancia de este tipo de iniciativas para los estudiantes. Por su parte, los profesores de la Cátedra de Producción colaboraron proponiendo aquellos proyectos con algunas de las características predefinidas: 
facilidad para emprender, impacto social, innovador o incorporación de elementos tecnológicos que diferencien de otros ya existentes.

La actividad se desarrolló el sábado 14 de mayo, 2016. Expusieron tres grupos de estudiantes acerca de los aspectos relevantes de los estudios de pre-factibilidad (mercado, técnico, organizacional, legal, financiero y evaluación del proyecto) que habían realizado para cada proyecto de la asignatura (535) y sus conclusiones sobre la importancia del proyecto. Los proyectos fueron: Fabricación de Jalea de Frijol, Batidos de frutas con base de leche no láctea (arroz, soya y almendra) y Club Diurno para Adulto Mayor "Edad de Oro". Los estudiantes presentaron sus proyectos con planos donde muestran las instalaciones previstas, otro grupo vestían camisetas con lo que sería el logotipo de su empresa, llevaron muestras para degustar. Cada grupo tuvo 30 minutos para exponer, el software utilizado para tal fin fue Power-Point y Prezzi, al final se hizo degustación de productos de dos de los proyectos. Se invitó para la jornada siguiente, a dos grupos de estos estudiantes, dada la calidad de sus proyectos y de sectores diferentes, Jalea de Frijol y Club Diurno "Edad de Oro", respectivamente Manufactura y Servicios.

\section{La primera Jornada Emprendedora}

Con la experiencia previa y la motivación que generó entre los estudiantes participantes, se decide planificar un evento mayor para el sábado 18 de junio del 2016. Participaron estudiantes de diversas regiones del país y se realiza en una fecha posterior a finalizar el cuatrimestre con el propósito de no distraer al estudiante en la preparación para sus pruebas escritas al final del período, asegurando así, la participación de los interesados.

Se seleccionaron proyectos acorde a las características previamente descritas en la jornada piloto, las calificaciones obtenidas no precisaba fuesen nivel muy bueno 0 excelencia, sino la idea de negocios del proyecto, así como la zona de residencia de los estudiantes. Entre los participantes había tanto estudiantes de la precitada asignatura (cód. 535), como una estudiante con sus trabajos finales de graduación (TFG) y un grupo multidisciplinario con un proyecto proveniente de otra iniciativa. 
Todos ellos exponiendo sus ideas de negocios relacionadas con la conformación de empresas o mejoras a una empresa existente (TFG).

\section{Estructura utilizada en las "Jornadas Emprendedoras"}

Se definió dos elementos para la primera jornada: el tiempo para exponer cada proyecto sería de 15 minutos (exposición 10 minutos y preguntas 5 minutos) y una "guía" donde se recoge información relevante que distinga cada proyecto. El esquema utilizado (ver figura 2) ayudó a estandarizar el contenido general a ser presentado. La información es obtenida del mismo trabajo desarrollado por los estudiantes participantes que cursaron la asignatura 535. Por ejemplo, en el proyecto "Exotic Jam" (jaleas combinadas con bajo contenido de azúcar), siguiendo la "guía" destacan el beneficio de la Generación de empleo, por ser originarios de Turrialba y las fuentes de empleo en la zona son escasas. Otro proyecto ("Jalea de frijol") resaltó su fortaleza de abrir oportunidades a los productores de frijol en su zona (esto lo indicaban dentro del esquema Proveedores), pues se les dificulta mucho la comercialización por exceso de oferta y menores precios del grano básico. Además plantean subcontratar a talleres metalmecánicos costarricenses, la fabricación de algunos equipos, creándose otra oportunidad señalada en el esquema bajo Tecnologías a utilizar. 
Figura 2. Guía de aspectos relevantes de proyectos a presentar en jornadas

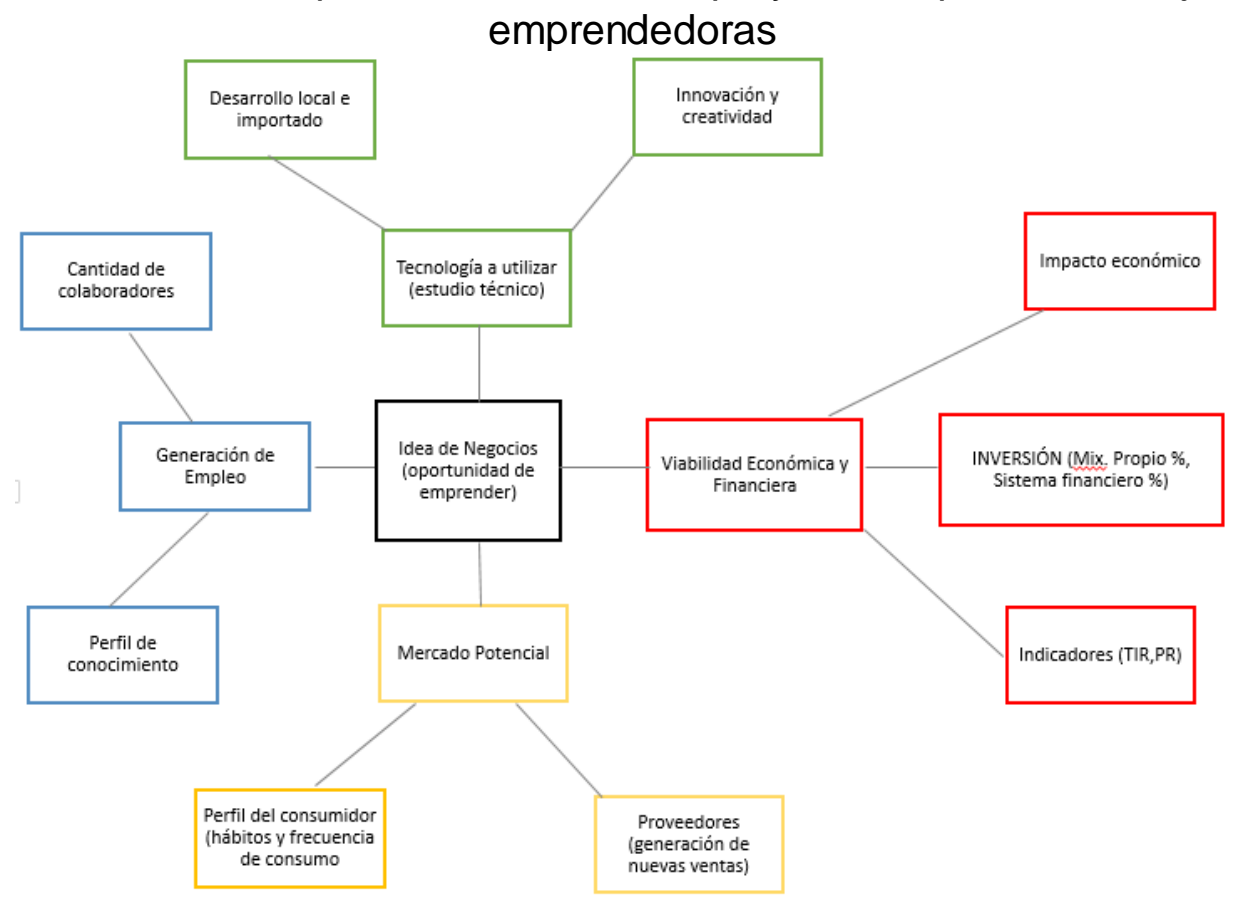

Fuente: Elaboración propia basado en los lineamientos que se le dan a los estudiantes

Vale mencionar que se indujo a los estudiantes el uso del software Popplet para montar ahí sus presentaciones que expondrán en la jornada. Se les dejó videos de YOU TUBE sobre utilizar dicho software: https://www.youtube.com/watch?v=WRjyfqa8VT4"

La agenda del evento se construyó considerando una plenaria y cada grupo contó con 15 minutos para exponer su proyecto. Esto facilitó escuchar cuatro proyectos por hora, y a las personas invitadas (empresarios y funcionarios del Registro Nacional) se les brindó 30 minutos para transmitir sus experiencias a los estudiantes.

En síntesis, la actividad se desarrolló a lo largo de un día entero, iniciando a las 9:00 y finalizando 17:00 horas con receso en el almuerzo. Se aprovechó para compartir de manera informal con un emprendedor que interactuó con los estudiantes, 
mientras estos degustaban su almuerzo. Las presentaciones de estudiantes se distribuyeron intercaladas con las charlas de emprendedores y otras personas invitadas. Se logró una agenda variada y del interés de los participantes, pues algunos empresarios lograron escuchar las iniciativas de los estudiantes. Los temas seleccionados para las charlas a los estudiantes tenían el objetivo de brindar conocimiento útil para el proceso de emprender un negocio. Las profesionales del Centro de Apoyo a la Tecnología y la Innovación del Registro Nacional, impartieron: "Marcas" y "Patentes". Los empresarios invitados eran emprendedores de sus propios negocios, sus tres charlas constituyeron una rica experiencia para los estudiantes. En términos generales, los gerentes relataron sus vivencias para lograr iniciar sus negocios, a la vez que compartieron aspectos claves de éxito en las empresas que ahora dirigen.

El aporte de la charla del señor Ricardo Rojas Soto (Gerente General) de Industrias de Huevo INNOVO, consistió en que la empresa rompe el paradigma y transforma el uso tradicional de la industria avícola, innovando con la producción y comercialización de huevo líquido para la demanda local en la industria de alimentos. Ubicada en el Cantón de Naranjo, que es una zona rural de la provincia de Alajuela, y a un año de operar, la empresa ha logrado posicionarse en el mercado.

Marco Escamilla es emprendedor y CEO de QUARZO INNOVACION, una empresa enfocada en desarrollar Apps, Software y soluciones en la nube para entidades de ahorro y crédito que está posicionada, en su nicho de mercado para Centroamérica, a la vanguardia de aplicaciones de tecnologías. En el conversatorio durante el almuerzo, Marco cuenta su experiencia como emprendedor e innovador en los desarrollos de aplicaciones. La charla fue muy motivadora y resaltando que cuando existe alguna idea de negocio, hay que tratar de plasmarla, esto conversando e intercambiando impresiones con los estudiantes.

Karen Leitón, emprendedora de Productos Cerro Blanco, empresa familiar, cuyo giro de negocios es el de productos lácteos (producción y comercialización) como: 
yogurt, quesos entre otros. Karen ocupa la posición de Gerente General y su charla transmitió la experiencia como emprendedora que sin saber nada sobre administración o el desarrollo de tecnologías de alimentos lácteos, incursiona en el mercado, logrando posicionar sus productos en supermercados de renombre en el país con la aceptación de los consumidores.

Cinthia Jiménez es Licenciada-egresada de la UNED en Mercadeo. Ella es Emprendedora de Almohadas Viva Mamá, que se especializa en artículos médicos para la lactancia materna. Su charla es el vivo ejemplo de una estudiante de la ECA que inicia su negocio por necesidades económicas para costear su tesis y obtener su título de licenciada.

La última charla fue la del profesor Luis Emilio Rodríguez, quien labora para la Cátedra de Banca y Finanzas (UNED), su exposición se concentró en aquellos espacios de apoyo que tienen los emprendedores para poder llevar a cabo sus negocios y donde pueden acudir para obtener financiamiento y acompañamiento.

\section{Metodología y vinculación externa}

En la figura 3 se muestran cómo se contextualizan el planteamiento del problema en estudio, este se basa en lo propuesto por (Hernández, Fernández \& Baptista, 2014). Es así, que se tratará de recopilar toda la información necesaria, y un cuestionario aplicado a los estudiantes. Se ubicó una cuota de proyectos para todo el país, buscando el apoyo de la Federación de Estudiantes para cubrir los gastos de viáticos para los estudiantes participantes. 
Figura 3. Proceso del planteamiento del problema

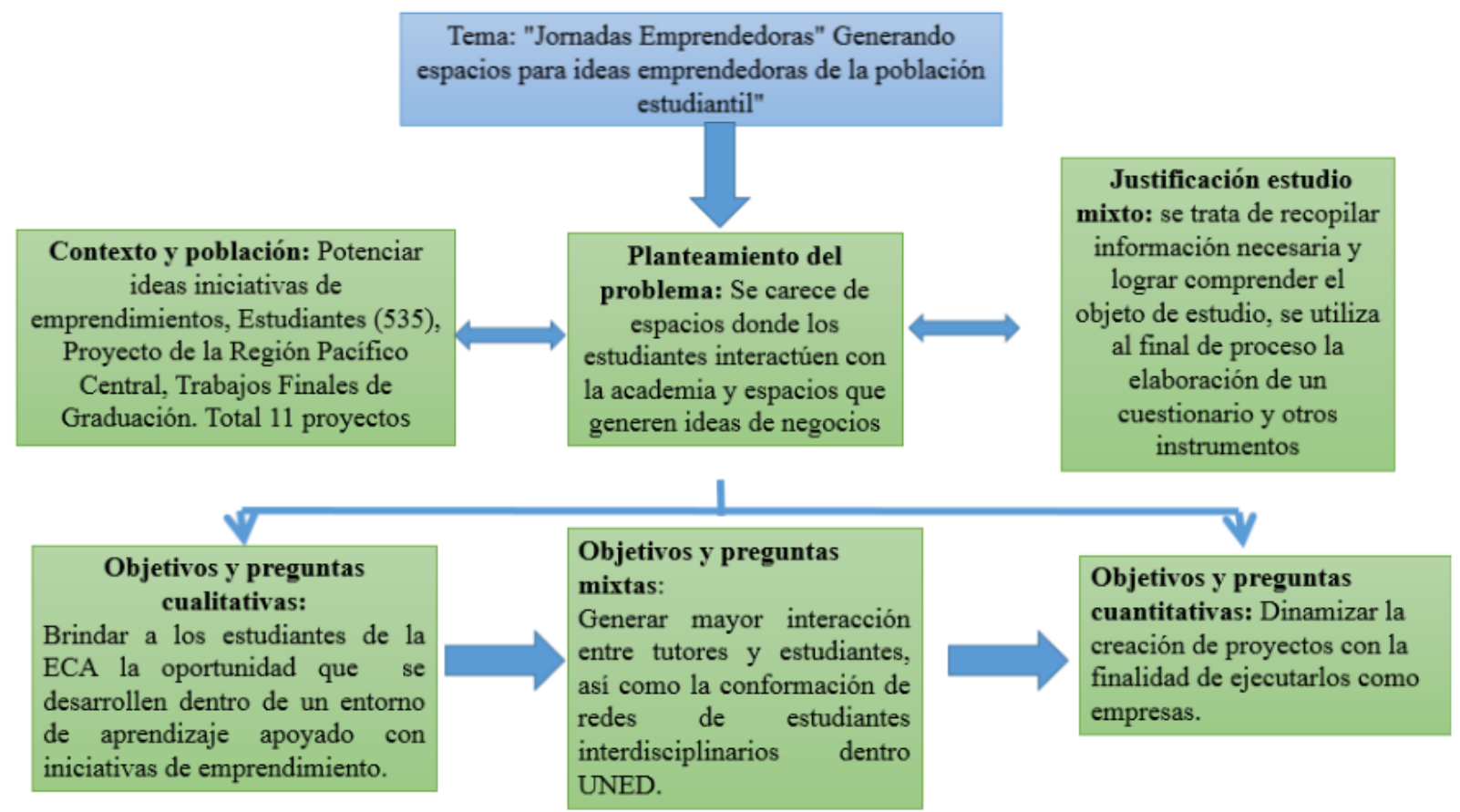

Fuente: Elaboración propia basada en el Proceso del planteamiento del problema según (Hernández, Fernández \& Baptista, 2014)

Asímismo, se realizaron llamadas para confirmar el interés real a participar para obtener finalmente una lista de 20 estudiantes de la asignatura (cód. 535) con sus trabajos a ser presentados. Se invitó a los Ministerios de Ciencia y Tecnología (MICT) y al de Economía y Comercio (MEIC) y municipalidades de donde los estudiantes son originarios. La idea es que funcionarios de dichas instituciones, escuchen las propuestas de negocios para crear esa aproximación y contacto en caso de interés mutuo estudiante-entidad y buscar hacia futuro generar alguna sinergia.

\section{Los proyectos participantes y su importancia}

La variedad de tipos de proyecto se resume en: productos alimenticios (7), Textil (1), Productos de Belleza (1), Artículos-Hogar (1) y Servicios (1). Bajo productos alimenticios, hubo siete diferentes tipos de alimentos y cada proyecto con sus propias particularidades que los hacía destacar y se pueden enumerar en la tabla 1. 
Tabla 1. Categoría de proyectos y sus atributos

\begin{tabular}{|c|c|c|c|c|}
\hline Categoría & Proyecto & Atributo & Zona & Carrera \\
\hline \multirow{7}{*}{$\begin{array}{l}\text { Productos } \\
\text { alimenticios }\end{array}$} & $\begin{array}{l}\text { Aderezos Las } \\
\text { Brumas, S.A. }\end{array}$ & $\begin{array}{l}\text { Integración con productores } \\
\text { locales, ingredientes orgánicos. }\end{array}$ & $\begin{array}{l}\text { Norte de } \\
\text { Cartago }\end{array}$ & $\begin{array}{c}\text { Administración Énfasis en: } \\
\text { Banca y Finanzas }\end{array}$ \\
\hline & $\begin{array}{l}\text { Cultivo de } \\
\text { Mejillón }\end{array}$ & $\begin{array}{l}\text { Emple o en zona deprimida, } \\
\text { Levantar la producción y } \\
\text { comercialización. }\end{array}$ & $\begin{array}{l}\text { Golfo de } \\
\text { Nicoya }\end{array}$ & $\begin{array}{l}\text { Multidisciplinario } \\
\text { (COMIEX) }\end{array}$ \\
\hline & Jalea de Frijol & $\begin{array}{l}\text { Integración con productores } \\
\text { locales y fabricantes-equipo }\end{array}$ & Orotina & $\begin{array}{l}\text { Administración Énfasis en: } \\
\text { Contabilidad }\end{array}$ \\
\hline & $\begin{array}{l}\text { Vinagretas } \\
\text { doña Virginia }\end{array}$ & $\begin{array}{l}\text { Integración productores } \\
\text { locales. }\end{array}$ & $\begin{array}{l}\text { Oreamuno } \\
\text { de } \\
\text { Cartago }\end{array}$ & $\begin{array}{l}\text { Administración Énfasis en: } \\
\text { Banca y Finanzas, } \\
\text { Contabilidad }\end{array}$ \\
\hline & $\begin{array}{l}\text { Naturals } \\
\text { Lunick }\end{array}$ & $\begin{array}{l}\text { Bajo contenido de azúcar, } \\
\text { integración productores locales }\end{array}$ & $\begin{array}{l}\text { Carrizal, y } \\
\text { Ciudad } \\
\text { Neilly }\end{array}$ & $\begin{array}{l}\text { Administración Énfasis en: } \\
\text { Contabilidad }\end{array}$ \\
\hline & $\begin{array}{l}\text { Ensaladas } \\
\text { acuapónicas }\end{array}$ & Innovación, e cológico. & $\begin{array}{l}\text { Alajuela y } \\
\text { San José }\end{array}$ & $\begin{array}{l}\text { Administración Énfasis en: } \\
\text { Banca y Finanzas, Negocios } \\
\text { Internacionales }\end{array}$ \\
\hline & Exotic Jam & $\begin{array}{l}\text { Bajo contenido de azúcar, } \\
\text { integración con productores } \\
\text { locales }\end{array}$ & Turrialba & $\begin{array}{l}\text { Administración Énfasis en: } \\
\text { Contabilidad }\end{array}$ \\
\hline Textil & SunFill & $\begin{array}{l}\text { Innovación (ropa con filtro } \\
\text { solar) para la salud física, } \\
\text { fuentes de empleo a ex } \\
\text { empleadas de antiguas maquilas. }\end{array}$ & Palmares & $\begin{array}{l}\text { Administración Énfasis en } \\
\text { Dirección de Empresas }\end{array}$ \\
\hline $\begin{array}{l}\text { Productos } \\
\text { de Belleza }\end{array}$ & Mi Jabonería & $\begin{array}{l}\text { Trabajo Final de Graduación } \\
\text { sobre estrategia para me jorar } \\
\text { empre ndimiento (empresa). }\end{array}$ & San José & $\begin{array}{l}\text { Administración Énfasis en: } \\
\text { Dirección de Empresas } \\
\text { (TFG) }\end{array}$ \\
\hline $\begin{array}{l}\text { Artículos- } \\
\text { Hogar }\end{array}$ & Rustiko, S.A. & $\begin{array}{l}\text { Ecológico utiliza tarimas } \\
\text { reciclando madera. }\end{array}$ & Palmares & $\begin{array}{l}\text { Administración Énfasis en: } \\
\text { Mercadeo }\end{array}$ \\
\hline Servicios & $\begin{array}{l}\text { Club Diurno } \\
\text { Adulto Mayor }\end{array}$ & $\begin{array}{l}\text { Social: necesidad del cuido de } \\
\text { adultos para que logren trabajar } \\
\text { sus familiares }\end{array}$ & San José & $\begin{array}{l}\text { Administración Énfasis en: } \\
\text { Negocios Internacionales }\end{array}$ \\
\hline
\end{tabular}

Fuente: Elaboración propia basado en los datos de los trabajos presentados por los estudiantes

En las otras categorías también hay atributos de distinta índole, entre sus aspectos: lo ecológico, la innovación, lo social y también aspectos de fuentes de empleo. La población estudiantil participante muchos es de zonas fuera de la Gran Área Metropolitana-GAM (ciudades de: San José, Cartago, Alajuela y Heredia) o zona urbana. Más adelante, en las tablas 2 y 3 , se divide para diferenciar lo rural de las localidades del GAM. Como parte de la selección de proyectos, también se pensó en lograr representación de estudiantes de zona rural y urbana. 
Tabla 2. Proyectos de fuera del GAM y su oportunidad respecto al Índice de Progreso Social (IPS)

\begin{tabular}{|c|c|c|}
\hline Proyecto (Atributo) & Zona & Comentarios \\
\hline $\begin{array}{l}\text { Aderezos Las Brumas, S.A. } \\
\text { (Integrar productores locales, } \\
\text { ingredientes orgánicos). }\end{array}$ & $\begin{array}{l}\text { Cartago: } \\
\text { Zona norte } \\
\text { IPS: } 78.08\end{array}$ & $\begin{array}{l}\text { El IPS es alto, se estima que se pueden generar } \\
\text { oportunidades a la inclusión de hogares con jefatura } \\
\text { femenina. Ventaja: oportunidad laboral hacia este sector. }\end{array}$ \\
\hline $\begin{array}{l}\text { Rustiko, S.A. } \\
\text { reciclando tarima }\end{array}$ & $\begin{array}{l}\text { Palmares } \\
\text { IPS: } 78.07\end{array}$ & $\begin{array}{l}\text { Posee un IPS alto, con mayores problemas en lo que es el } \\
\text { rezago escolar, tasa de obesidad, y tasa de mortalidad por } \\
\text { enfermedades no contagiosas. Por lo cual las oportunidades } \\
\text { se pueden enfocar como solventar estos problemas. }\end{array}$ \\
\hline $\begin{array}{l}\text { ExoticJam (Bajo contenido } \\
\text { de azúcar, integración con } \\
\text { productores locales) }\end{array}$ & $\begin{array}{l}\text { Turrialba } \\
\text { IPS: } 75.61\end{array}$ & $\begin{array}{l}\text { Presenta IPS, alto, respecto a los que se presentan en GAM, } \\
\text { aunque existe oportunidades con relación a matricula en } \\
\text { educación primaria, muertes por enfermedades infecciosas, } \\
\text { madres adolescentes e inclusión de la población indígena }\end{array}$ \\
\hline $\begin{array}{l}\text { Jale a de Frijol }(\mathrm{I} \\
\text { con productores } \\
\text { fabricantes-ec }\end{array}$ & $\begin{array}{c}\text { Orotina } \\
\text { IPS: } 70.14\end{array}$ & $\begin{array}{l}\text { Oportunidades que se pueden originar, deberán enfocarse a } \\
\text { la población escolar, ya que existe un rezago, y una } \\
\text { cantidad de jóvenes 'Nini", que ni trabajan, ni estudian, no } \\
\text { generan ninguna fuente de ingreso al hogar. }\end{array}$ \\
\hline $\begin{array}{l}\text { Cultivo de Mejillón } \\
\text { (Empleo en zona deprimida, } \\
\text { levantar la producción y } \\
\text { comercialización). }\end{array}$ & $\begin{array}{l}\text { Golfo de } \\
\text { Nicoya } \\
\text { (Puntarenas } \\
\quad \text { ) } \\
\text { IPS: } 69.75\end{array}$ & $\begin{array}{l}\text { Las zonas de puertos generan un rezago social aún más } \\
\text { grande que otros cantones, hay mayores oportunidades por } \\
\text { poco acceso a la matrícula de educación secundaria y la } \\
\text { cantidad de madres adolescentes. El proyecto tiene el } \\
\text { propósito de generar fuentes de empleo a familias } \\
\text { completas en producir y comercializar el mejillón. }\end{array}$ \\
\hline $\begin{array}{l}\text { Naturals Lunick (Bajo } \\
\text { contenido de azúcar, } \\
\text { integración productores } \\
\text { locales) }\end{array}$ & $\begin{array}{l}\text { Ciudad } \\
\text { Neilly } \\
\text { (Corredore) } \\
\text { IPS: } 69.17\end{array}$ & $\begin{array}{l}\text { En el Cantón Corredores, el IPS dentro del promedio } \\
\text { respecto a los otros, aquí las oportunidades son diferentes } \\
\text { que en otros regiones. Las mismas están enfocadas hacia } \\
\text { una población analfabeta, se debe incluir al adulto mayor, a } \\
\text { una población con discapacidad, y a poblaciones indígenas. }\end{array}$ \\
\hline
\end{tabular}

Los estudiantes de zona rural demostraron estar muy conscientes de la importancia de proyectos que generan fuentes de empleo en sus zonas, a lo largo de sus exposiciones hacían alusión a ese beneficio para sus comunidades donde las opciones son pocas. Lo anterior es típico de muchos países en Latinoamérica que industria y comercio están en zonas urbanas.

Es la primera actividad a lo interno de la ECA, donde intervienen tres actores Universidad-Institución (Privada o Estatal)-Estudiantes, todos integrados en una sola actividad para la creación de empresas. Este escenario es muy novedoso a lo interno de la ECA y de UNED. Es muy importante lograr sistematizar esa integración para explorar oportunidades de crear emprendimientos con impactos positivos, sobre todo en zonas donde se está necesitando ampliar las fuentes de 
empleo, así como apoyar encadenándose a pequeños empresarios o talleres que brindan servicio a otras industrias. Además, el estudiante fortalece su capacidad de auto-emplearse, al poner en práctica sus habilidades desarrolladas y conocimientos construidos a lo largo de su carrera, contextualizándolo con su entorno.

Para efectos de análisis sobre el potencial de cada proyecto, se comparan algunas zonas, donde residen los estudiantes con su propuesta, y el Índice de Progreso Social (IPS). Se logra apreciar (ver Tablas 2 y 3) las oportunidades de apoyo en la zona que favorecería la idea de negocio de hacerlo realidad y de ahí la importancia de emprender. En la tabla 2, se observa que la mayoría de proyectos participantes fueron de zona rural, y no precisamente por tal razón son los de menor IPS, ya que hay también organización de sociedad civil muy proactivas como la de Palmares que trata de invertir sus recursos en bienes de la comunidad (parques, escuelas y otros centros de apoyo social). Vale mencionar que hay otras zonas donde el IPS es mucho menor de los que aparecen en tabla 2, sin embargo, no hubo participación de estudiantes de dichas zonas en las jornadas de Junio.

En la tabla 3 se agrupan proyectos de estudiantes de la zona urbana y cuyos IPS son de valores aceptables. También hay cantones de la zona urbana con esos valores bajos, pero no es el fin del presente estudio el análisis a detalle, sino más bien resaltar que al gestionar y apoyar a estudiantes, donde se les brinde un ambiente de emprendimientos, puede hacer la diferencia en la educación a distancia. Este tipo de espacios académicos abiertos que apunten al emprendimiento o creación de empresas entre la población estudiantil, tiene la posibilidad de aportar soluciones al desempleo en las comunidades donde los estudiantes residen.

Es momento de iniciar con el desarrollo de actividades que coadyuven a la población estudiantil de la UNED a forjar sus competencias, la creatividad y la innovación. Es preciso que los estudiantes pasen por la experiencia de trabajar colaborativamente en un ambiente de emprender su propio negocio. Respecto al término de competencias hay autores que mencionan su importancia como parte de 
la formación integral en los procesos de aprendizaje-enseñanza de los estudiantes, así lo señala Santandreu-Canós-Marín (2015) citando a Delgado et al., 2016 en su artículo para la revista educade.

Tabla 3. Proyectos dentro del GAM y su oportunidad respecto al Índice de Progreso Social (IPS)

\begin{tabular}{|c|c|c|c|}
\hline Proyecto (Atributo) & Zona & IPS & Comentarios \\
\hline $\begin{array}{l}\text { Club Diurno Adulto } \\
\text { Mayor (Social: } \\
\text { necesidad del cuido de } \\
\text { adultos para que logren } \\
\text { trabajar sus familiares) }\end{array}$ & $\begin{array}{c}\text { San José } \\
\text { (San Pedro, } \\
\text { Montes de } \\
\text { Oca) }\end{array}$ & 76.08 & $\begin{array}{l}\text { Por ser cantones que pertenecen al GAM (Gran área } \\
\text { metropolitana), tienen otras necesidades diferentes que } \\
\text { los cantones alejados, presenta áreas de oportunidades } \\
\text { que se pueden desarrollar, por ejemplo, muertes por } \\
\text { enfermedades no contagiosas, matrícula de educación } \\
\text { primaria y secundaria, tasa de muertes por IRA }\end{array}$ \\
\hline $\begin{array}{l}\text { Ens aladas acuapónicas } \\
\text { (Innovación, ecológico) } \\
\text { Mi Jabone ría (TFG) } \\
\text { sobre estrategia para } \\
\text { mejorar emprendimiento } \\
\text { de una empresa). }\end{array}$ & $\begin{array}{l}\text { Alajuela y } \\
\text { San José }\end{array}$ & $\begin{array}{c}73.78 \\
70.4\end{array}$ & $\begin{array}{l}\text { Por ser cantones que pertenecen al GAM (Gran área } \\
\text { metropolitana), tienen otras necesidades diferentes que } \\
\text { los cantones alejados, presenta áreas de oportunidades } \\
\text { que se pueden desarrollar, como por ejemplo, muertes } \\
\text { por enfermedades infecciosas, matrícula de educación } \\
\text { secundaria, y cobertura forestal. }\end{array}$ \\
\hline
\end{tabular}

Fuente: Elaboración propia basado en los datos de los trabajos presentados por los estudiantes

\section{Resultados}

La incorporación de estudiantes de diferentes centros universitarios y carreras que generen potenciales soluciones a sus comunidades fue exitosa. Se demuestra interés, tanto por los centros universitarios de zona rurales, como por parte de los estudiantes en participar. Un primer acercamiento entre los estudiantes, universidad, empresas, entidades estatales que apoyan el emprendimiento o innovación dentro de las universidades. Luego, también a lo largo del proceso se logra la participación tanto de los tutores como estudiantes, por medio de los proyectos seleccionados para enfocarse hacia potenciales emprendimientos.

Los estudiantes obtuvieron conocimientos, motivación y acercamiento a aspectos importantes para emprender una idea de negocio. También se brindó asesoría técnica al estudiantado para plasmar estas ideas de negocios. Se cumplió con la ejecución de lo previsto, al haber realizado dos Jornadas Emprendedoras conforme lo previsto en el presupuesto operativo académico POA-2016. Se puede resaltar el interés por parte de los estudiantes, no sólo aprobar la asignatura, sino interés por agregar conocimiento haciendo otro tipo de actividades, que si bien es cierto son 
académicas, demandan otro tipo de habilidades, que por lo general un estudiante de la UNED, no está acostumbrado a realizar.

El hecho de que el estudiante pasó por la experiencia de exponer ante un público, bajo la presión del tiempo (10 minutos), se logra corroborar la capacidad de cada uno de los estudiantes participantes, al realizar una actividad poco frecuente por el modelo a distancia y que son competencias importantes para un administrador. La creación de todo ese ambiente emprendedor: las 11 ideas de negocios que son compartidas entre los mismos estudiantes, los conocimientos y experiencias de emprendimientos por parte de los empresarios que transmiten a la población estudiantil; son también logros positivos.

Adicionalmente, las charlas del Registro Nacional y el profesor que enfatiza en aspectos claves para el inicio de toda empresa, son elementos fundamentales para apoyar ese ambiente de emprendedurismo. Lo anterior ayuda a pasar de una idea de negocios a una empresa, considerando la visón de la realidad costarricense de los emprendedores y sus rasgos para poder sacar adelante sus empresas. Respecto a potenciar las ideas de negocios, se logró confirmar, el hecho que los estudiantes pueden lograr ser innovadores y capaces generar emprendimientos.

Se observó proyectos que pueden categorizarse bajo los tres enfoques característicos de emprendimientos: social, ecológico y empresas de base tecnológica. Ejemplo de estos fueron el Club Diurno Adulto Mayor (Liberia y San José) que tiene un potencial de un emprendimiento social, Rustiko, S.A. y Ensaladas Acuapónicas con aspectos ecológicos para ser impulsados como emprendimientos de base ecológica, por impulsar un desarrollo más sostenible en sus propias comunidades.

Por otra parte, el proyecto de Jalea de Frijol, si bien nace de la receta casera de la abuelita de los estudiantes integrantes del grupo, el proceso de producción a escala industrial que pretenden desarrollar el proyecto requiere una serie de inversiones en elementos tecnológicos, tanto por la maquinaria como en el proceso de 
transformación de los alimentos, para lograr una vida útil mayor a la que podrá tener la producción a nivel artesanal.

\section{Encuesta}

A menos de una semana posterior a la actividad, se realizó una encuesta para conocer la opinión de los estudiantes acerca de sus experiencias con la iniciativa. La encuesta fue respondida por la mitad de estudiantes participantes, el perfil demográfico y laboral se resume en tabla 4. No se discutirán todos los ítems de dicho documento, se comentarán los principales acordes a los objetivos planteados. Llama la atención respecto al tipo de trabajo, siendo el $70 \%$ que lo hace en un negocio propio o familiar, así como el hecho que el $80 \%$ está en una empresa compuesta de 1-5 empleados que podemos decir es en una microempresa.

Tabla 4. Perfil de encuestados

\begin{tabular}{|l|l|l|l|}
\hline Género & Rango edad & Tipo de trabajo & Tamaño empresa \\
\hline Femenino: $\mathbf{9 0 \%}$ & 26 a $40 \rightarrow 80 \%$ & Propio, familiar:70\% & 1 a 5 empleados $\rightarrow$ 80\% \\
\hline Masculino: $\mathbf{1 0 \%}$ & 18 a $25 \rightarrow 20 \%$ & Asalariado: 30\% & 11 a $20 \rightarrow 10 \%$, más de 20 $\rightarrow 10 \%$ \\
\hline
\end{tabular}

Entre los principales resultados está la calificación general al evento que en una escala de Likert (1 a 5), donde "5" es "Excelente" y el "1" "Mala", arrojó el valor promedio de "4.2" o sea que la consideran "Muy buena" y sobre si recomendaría a otros la actividad, utilizando escala de Likert, donde "5" es "Totalmente de acuerdo" y el " 1 " "Totalmente en desacuerdo" se indicó en $90 \%$ de los casos un " 5 " y $10 \%$ un "4".

Muy importante mencionar el hecho que a la pregunta: ¿Participaría en una "incubadora de negocios"? la respuesta fue que sí en un $100 \%$ y a la pregunta con escala de Likert (1 a 5): ¿considera conveniente que en UNED se implemente una iniciativa de "incubadora" con los estudiantes? Un 80\% indicó estar totalmente de acuerdo (5) y un $20 \%$ estar de acuerdo (4). 
Se preguntó si les pareció apropiada la metodología utilizada en la presentación de los proyectos, a lo cual en un $90 \%$ dijeron que Sí. También se solicitó la mención de aspectos a resaltar de la actividad, además de resaltar repetidas veces las charlas, algunas otras de las respuestas fueron: "Testimonios de empresarios"/ "Promoción de los negocios, generación de ideas empresariales e innovación"/ "El enfoque de negocios"/ "Excelentes proyectos y muy útiles las charlas que dieron"/ "Lo motiva a uno para poder llevar a cabo el proyecto".

Otra de las preguntas fue sobre las seis charlas que recibieron, se les consultó acerca de la que consideran más útil para sus intereses de emprender, respondieron con un peso del $30 \%$ para el conversatorio con Marco Escamilla y el tema de Marcas. Además, se preguntó acerca de la enseñanza que dejaron las charlas y las respuestas fueron variadas y a continuación se citan algunas: "Nunca rendirse y buscar hasta la última opción para seguir adelante"/ "Orientación de como emprender un negocio"/ "Fue muy provechoso, se presentaron ideas de negocios muy buenas, además de que se motivó mucho a los invitados a ser emprendedores y les dieron charlas importantes para continuar con sus proyectos"/ "En realidad todas fueron provechosas, dejan enseñanzas como que vale la pena esforzarme por emprender un negocio y que en el camino siempre hay obstáculos pero con perseverancia y esfuerzo se pueden superar"/ "El proceso de la constitución legal del proyecto innovador que presentamos"/ "Superación personal, organización empresarial y desarrollo empresarial."

Para efecto de tomar acciones hacia futuro, se realizaron las siguientes tres preguntas que se irán exponiendo seguidas de sus respuestas. ¿Tiene alguna expectativa por parte de la ECA (UNED) para colaborar con su proyecto? ¿Indique cuál? Las respuestas: "Si. Asesorías, retro-alimentación para mejorar"/ "Recomendaciones para introducir el producto"/ "Capacitación en el área administración"/ "Que brinde más apoyo y más charlas"/ "Generar contactos para buscar financiamiento para los proyectos"/ "Ayuda en el área de mercadeo, para el producto/ Guía para emprender el negocio". 
¿Qué tipo de apoyo le interesaría recibir para continuar su idea de proyecto? Respuestas: "Financiamiento"/ "Orientación en inicio de un negocio específico de servicios profesionales"/ "Asesorías de todo tipo referente a crear una empresa"/ "Generar contactos para buscar financiamiento para los proyectos"/ "Ayuda técnica en el área de alimentos".

¿Considera importante y oportuno que la ECA (UNED) brinde algún tipo de asesoría? Indique qué tipo de asesoría. Respuestas: "Legal y técnica"/ "Orientación financiera y proceso"/ "Método de emprendedurismo de un negocio, servicios profesionales, banca de desarrollo"/ "Asesoría financiera"/ "Las entidades e instituciones que puedan dar asesoría, para el tipo de proyecto"/ "PYMES".

Se observa en varias de estas respuestas, la motivación generada en los estudiantes y seguro les genera un mayor interés en el aprendizaje sobre esos temas. La atención de participantes en conversatorio del almuerzo era alta, hubo mucha interacción con el expositor y estudiantes, siendo esta una de las charlas preferidas. Aquí vale citar que según Lamas Rojas (2008) "cuando el estudiante se enfrenta a la tarea del aprendizaje de un contenido escolar lo hace de modo que en esta actividad participan tanto las variables propiamente cognitivas y motivacionales, como las afectivas." (p.17). Por otra parte, se conoce que “...un alumno eficaz en sus procesos de pensamiento auto-regula su conducta aunque no sólo cognitivamente sino también desde su vertiente motivacional." (p.18)

\section{¿Qué sigue?}

Hacia futuro se prevé que continúen participando estudiantes que se matriculen en la asignatura código 535, a lo largo de varios períodos académicos. Lo anterior considerando que en dicha asignatura los tópicos se estudian desde un enfoque de proyecto de inversión, según Baca (2013) "es un plan que, si se le asigna determinado monto de capital y se le proporcionan diversos insumos, producirá un bien o un servicio útil a la sociedad". (p.2). También se espera que participen estudiantes con TFG, cuya temática sea algún estudio de factibilidad o bien que tenga potencial a convertirse en un emprendimiento. 
Se tiene previsto establecer para la próxima Jornada Emprendedora, a realizarse en Diciembre 2016, una preselección de proyectos en distintas categorías considerando los enfoques: Social, Ecológico (o Sostenible), Tecnológico y Encadenamientos. Se realizará un concurso para elegir uno por categoría y además se elegirá un ganador en categoría general. Además, se vinculará previamente, estudiantes con algunas municipalidades que estén interesadas en apoyar los proyectos según las zonas y comunidades donde residen los estudiantes o bien según localización de los proyectos.

\section{Conclusiones}

Como resultado de esta experiencia, se logra vislumbrar a corto plazo, contando con el apoyo por parte de las autoridades universitarias y la asignación presupuestaria respectiva, realizar y obtener un análisis más cercano a las realidades de una población por zonas geográficas con necesidades específicas y que se puedan vincular con instancias regionales, para hacer posible el hecho de enfocarse en las oportunidades, según la región y lo que conlleva este tipo de actividades académicas.

Es preciso promover la participación de personas provenientes de la comunidad, donde están los distintos Centros Estudiantiles Universitarios (CEU), y se pueda solicitar, por medio de dichos CEU's, la asistencia para que se les elabore algún estudio de pre-factibilidad o factibilidad (dependiendo el nivel requerido) con necesidades de algún financiamiento a solicitar a entidades bancarias.

Considerando la característica de lo multidisciplinario de los emprendimientos, pueden incluirse también aquellas otras unidades académicas (incluyendo las otras tres escuelas de UNED) que muestren interés en participar. En la universidad se cuenta con la Escuela de Ciencias Exactas y Naturales (ECEN), la Escuela de Ciencias Sociales y Humanidades (ECSH) y la Escuela de Ciencia de la Educación (ECED). El fin es involucrar estudiantes avanzados en el bachillerato de una carrera, y vincularlos con la realidad de una creación de idea de negocio, por un lado, y por otro, es el acercamiento, práctico y guiado por parte de una cátedra 
(profesores) que de manera voluntaria, se guía y se les dan herramientas, que en una asignatura por el tipo de evaluación con que se cuenta, a veces no es considerada.

Las Jornadas Emprendedoras ayuda en motivar a los estudiantes por solo el hecho de tomarles en cuenta que participen. Ellos se identifican con la actividad y se logra que exista un esfuerzo adicional más allá de lo académico, lo cual refuerza al estudiante respecto al valor de lo que implica estudiar. Además, rescatar ese interés de ser escuchado, de ser visible, donde lo cognitivo se mezcla con lo emotivo.

En el tema de estrategia de aprendizaje vale la pena establecer actividades como la Jornada Emprendedora para reforzar los conocimientos, capacidades y habilidades del profesional formándose en las ciencias administrativas. Parte importante de la calidad en educación superior es el brindar al estudiante que se forma, la oportunidad de poner en ejercicio sus competencias encarando el mundo de los negocios, así como trabajo en equipo, acercamiento a empresarios y ampliar su red de contactos, para que puedan tener otros referentes del entorno fuera del mundo académico donde está inmerso.

Se pretendió alinear la búsqueda de una meta y objetivo en común, que es lo perseguido por el trabajo colaborativo. Lo cual se obtuvo a través de la participación por parte de los estudiantes, que lograron realizar equipos de trabajo, aunque de regiones diferentes, las herramientas tecnológicas que existen en la actualidad ayudan de manera positiva este tipo de acercamientos, tanto estudiante-estudiante, como estudiante-profesor, estudiante-encargado de cátedra.

Los pares evaluadores que han visitado la ECA refieren necesidad de actividades donde se integren estudiantes-docente-empresas, y parte de los objetivos de las Jornadas Emprendedoras es facilitar un ambiente, en el cuál los estudiantes puedan integrarse de alguna manera con esos otros actores (docente y empresario). 
La actividad está abierta para conformar equipos multidisciplinarios, o bien que otros estudiantes de carreras variadas participen y durante el desarrollo propio de la jornada, se compartan experiencias y perspectivas diferentes, enfoques nuevos que dará otra visión al estudiante que podría tener saberes distintos.

Existen oportunidades claras para la ECA de establecer un programa de extensión sistematizada que sea ofrecido para que participen los estudiantes interesados en dar seguimiento a sus ideas de negocios expuestas en las Jornadas Emprendedoras y de esa manera lograr satisfacer las expectativas que genera este tipo de actividades. También se ha de gestionar la vinculación de los estudiantes participantes con las instituciones externas que brindan apoyo en aspectos de formación o económicos, a los potenciales emprendedores.

Hay claras oportunidades para UNED en integrar distintas unidades como escuelas y centros universitarios para alinear esfuerzos en concretar la generación de proyectos, que respondan a necesidades en las comunidades donde la universidad puede articular distintos actores para el apoyo y concretar un bien o servicio final de utilidad a la sociedad.

El uso del IPS puede servir de referencia muy útil para jerarquizar la asignación de recursos académicos en aquellas zonas donde dicho indicador arroje mayores oportunidades de resolver o aportar soluciones a los problemas existentes que estén al alcance de la universidad. Se detecta interés entre la población estudiantil acerca de la incubadora de negocios, habiendo indicios de participación por parte de estudiantes motivados con actividades que propicien el pertinente ambiente emprendedor.

En resumen, es viable incentivar el desarrollo de emprendimientos en estudiantes de UNED. Así como poder dinamizar entre estos, la creación de proyectos con la finalidad de ejecutarlos como empresas. A los estudiantes de la ECA se les puede brindar la oportunidad que se desarrollen en un entorno de aprendizaje apoyado con iniciativas de emprendimiento. Donde las cátedras son un catalizador clave para 
impulsar una mayor interacción entre tutores y estudiantes. UNED tiene todo el potencial de conformar redes de estudiantes interdisciplinarios por medio de un trabajo articulado de las escuelas y centros universitarios.

\section{Referencias}

Araya, V. (2013). Emprendedurismo Social. Costa Rica: ULACIT. Recuperado de: http://bb9.ulacit.ac.cr/tesinas/Publicaciones/045712.pdf

Baca-Urbina, G. (2013). Evaluación de Proyectos. México: Mc-Graw Hill

Cabello, C.R. (2006). Emprendedurismo dentro del ámbito universitario. Resultados obtenidos en una investigación hecha a 50 estudiantes de educación superior emprendedores-empresarios que desarrollan sus ideas dentro del campus universitario. In Morea, Francisco y Fornoni, Mariel, (Eds.), La formación de emprendedores como clave para el desarrollo (pp.179-202). Mar del Plata: Suárez; UNMdP. ISBN 987-1314-17-5 Recuperado de http://nulan.mdp.edu.ar/708/1/00365.pdf

Centro Latinoamericano para la Competitividad y el Desarrollo Sostenible (CLACDS) (2016). Costa Rica Propone, "Índice de progreso social" [online]. INCAE. Recuperado de http://www.costaricapropone.go.cr/acerca-de-ips

Druker, P. (1985). Innovation and Entrepreneurship. Elsevier Ltd. Estados Unidos, Recuperado de https://books.google.co.cr/books?id=1y331zUzhTEC\&pg=PR4\&dq=innovation +and+entrepreneurship+drucker+Elsevier\&hl=es\&sa=X\&ved=0ahUKEwi0040 K80XPAhWPuB4KHQX0DyYQ6AEILTAA\#v=onepage\&q\&f=false

De Mello, J. y Etzkowitz, H. (2003). The rise of a triple helix culture. International Journal of Technology Management \& Sustainable Development; 2(3), 159171.

Delgadillo, J. y Alburquerque, F. (2010). Emprendimientos de base ecológica, un modelo de interacción económica y territorial en Áreas Naturales y Protegidas de España y México. [online]. $1^{\text {a }}$ ed., Instituto de Investigaciones Económicas-UNAM, Universidad de Sevilla, Colegio de Tlaxcala, México, p.123. Recuperado de http://www.scielo.org.mx/pdf/prode/v43n169/v43n169a11.pdf

Etzkowitz, H. y Klofsten, M. (Jun 2005). The innovation region: toward a theory of knowledge-based regional development. $R \& D$ Management, 35(3), 243255. 
Hernández, R., Fernández, C. \& Baptista, P. (2014). Metodología de la Investigación, 6a ed. México: Ed. McGraw-Hill.

Lamas, H. (2008). Aprendizaje autor regulado, motivación y rendimiento académico. Revista de Psicología. Liberabit, 14(14), 15-20. Recuperado de http://www.scielo.org.pe/scielo.php?pid=S1729$\underline{48272008000100003 \& \text { script=sci arttext }}$

March, I. y Mora, R. (2007). C Creación de empresas de base tecnológica: factores de éxito y fracaso. Revista de Contabilidad y Dirección, Vol. 5, año 2007, pp. 97-120. http://www.accid.org/revista/documents/RCD5 castellano 97.pdf

Merino, C. y Villar, L. (2007). Factores de éxito en los procesos de creación de empresas de base tecnológica, [online]. Madrid: Universidad Autónoma de Madrid. Recuperado de https://repositorio.uam.es/bitstream/handle/10486/668659/EmpresasTecnol\% C3\%B3gica Merino El 2007.pdf?sequence=1

Santandreu, M. (2015). Fomentando la multidisciplinariedad y el trabajo en equipo (metodologías participativas): creación de empresas, una asignatura para cuatro grados. [online]. Universitat Politècnica de València, Escuela Politécnica Superior de Gandía. Recuperado de: https://www.google.es/search?hl=es\&q=Santandreu-Can\%C3\%B3sMar\%C3\%ADn\#hl=es\&q=Santandreu-Can\%C3\%B3sMar\%C3\%ADn+educade

Universidad Estatal a Distancia. (2004). Modelo Pedagógico. San José, Costa Rica: Editorial EUNED.

Universidad Estatal a Distancia. (2014). Plan Estratégico de la ECA, 2015-2020, Costa Rica

Universidad Estatal a Distancia, CIEl. (2016). Estadísticas de matrícula primer cuatrimestre 2016" [online]. Costa Rica: Disponible en:

http://www.uned.ac.cr/viplan/images/ciei/Informe de matr\%C3\%ADcula I cu atrimestre 2016.pdf

Universidad Estatal a Distancia, Cátedra de Producción. (2016). Orientaciones académicas para la asignatura Preparación y Evaluación de Proyectos 1er cuatrimestre; UNED

Zemler, A. W. (2008). Definiciones: "Emprendedurismo Social" [online] J. Marchers Inc. Comunicaciones para el Desarrollo Social. Disponible en http://bb9.ulacit.ac.cr/tesinas/publicaciones/045712.pdf 